1 Hacettepe Journal of Mathematics and Statistics

$\bigcap$ Volume 47 (3) (2018), $521-538$

\title{
Existence of periodic solutions for a mechanical system with piecewise constant forces
}

\author{
Duygu Aruğaslan*† and Nur Cengiz ${ }^{\ddagger}$
}

\begin{abstract}
In this study, we consider spring-mass systems subjected to piecewise constant forces. We investigate sufficient conditions for the existence of periodic solutions of homogeneous and nonhomogeneous damped spring-mass systems with the help of the Floquet theory. In addition to determining conditions for the existence of periodic solutions, stability analysis is performed for the solutions of the homogeneous system. The Floquet multipliers are taken into account for the stability analysis [3]. The results are stated in terms of the parameters of the systems. These results are illustrated and supported by simulations for different values of the parameters.
\end{abstract}

Keywords: Generalized piecewise constant forces, spring-mass system, stability and periodic solutions.

2000 AMS Classification: $34 \mathrm{~A} 36,34 \mathrm{~K} 13,34 \mathrm{~K} 20,37 \mathrm{~N} 05$

Received : 17.10.2016 Accepted : 20.04.2017 Doi : 10.15672/HJMS.2017.469

\section{Introduction}

In the literature, many mathematical models have been suggested to examine real world processes. In some cases, state of the process in previous time influences the present and future states of the process significantly. Thus, earlier suggested models have been improved by taking time delays into consideration. These models including some of the past states of the processes are expressed by delay differential equations. These equations are very important in various problems of economics, ecology, biology, engineering, medicine and physics $[17,20]$.

\footnotetext{
*Department of Mathematics, Süleyman Demirel University, 32260, Isparta, Turkey, Email : duyguarugas lan@sdu.edu.tr

$\dagger_{\text {Corresponding Author. }}$

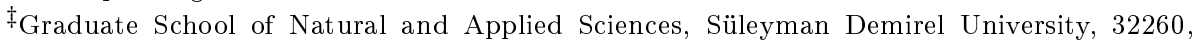
Isparta, Turkey,

Email : nurcengiz90@gmail.com
} 
Delay differential equations are generally given in the following form

$$
x^{\prime}(t)=f(t, x(t), x(t-\tau)),
$$

where $\tau$ is a positive delay value, $t \in \mathbb{R}, x \in \mathbb{R}^{n}$. It is clear that evolution of the system (1.1) depends both on the present and the previous time. If the greatest integer function $[t]$ instead of $t-\tau$ is taken as deviating argument in equation (1.1), then differential equations with piecewise constant argument of the form

$$
x^{\prime}(t)=f(t, x(t), x([t])),
$$

are obtained. Differential equations with piecewise constant argument can be considered in the class of delay differential equations since the rate of change of the system is determined by both the present value and the previously memorized values of the state variable $x$. This class of differential equations was described by Cooke and Wiener at the beginning of the 1980s [15]. In recent years, several studies about their theory such as existence and uniquness of solutions [15, 29], oscillations [1, 16, 26, 30, 31], existence of periodic and almost periodic solutions [1, 24, 27, 32], integral manifolds [23] and applications in the fields of biology, control theory, neural networks and biomedical models of disease $[9,10,11,12,19,21,22,28,33]$, stability [6, 7] are available. This type of equations has been usually examined by reducing them into discrete equations and applying numerical methods [11, 12, 15, 16, 17, 18, 22, 25, 29].

In (1.2), the distance between the switching moments is fixed as 1 unit. Taking any piecewise constant function instead of the greatest integer function, differential equations with piecewise constant argument were generalized by Akhmet in [4]. Later, differential equations with piecewise constant argument of generalized type have been studied by many authors $[2,5,6,8,11,13,14]$.

A classical homogeneous damped spring-mass system is given by

$$
m \ddot{x}+c \dot{x}+k x=0,
$$

where $x(t)$ is the displacement of the mass, $m>0$ is the mass, $c>0$ is the damping coefficient and $k>0$ is the spring constant. This system exhibits damped harmonic motion. In other words, if friction force acts on an oscillating system, then the oscillation amplitude approaches to zero. According to the sign of the discriminant

$$
\Delta=\frac{c^{2}}{m^{2}}-4 \frac{k}{m}
$$

of the characteristic equation

$$
r^{2}+\frac{c}{m} r+\frac{k}{m}=0,
$$

the behavior of the system is determined. If $\Delta>0, \Delta=0$ and $\Delta<0$, then the system exhibits motion with over damped, critical damped and under damped, respectively. The motions with over damped and critical damped are not oscillating, but the motion with under damped is oscillating. If this system has an external force $F$, it is described as follows

$$
m \ddot{x}+c \dot{x}+k x=F .
$$

In [16], taking the greatest integer function as deviating argument for the force and using the method of reduction to discrete equations, Dai and Singh studied the oscillatory motion of the following spring-mass system

$$
m \ddot{x}+c \dot{x}+k x=A x([t]),
$$

where $A$ denotes the magnitude of the force and the last system has discontinuities at the moments $[t]$ because of the piecewise constant force $F=A x([t])$. 
In this study, we take external forces with piecewise constant argument of generalized type instead of greatest integer function for the damped spring-mass system. We determine sufficient conditions for stability of solutions of the linear homogeneous system using Floquet multipliers. Additionally, we deal with existence and uniqueness of periodic solutions for both homogeneous and nonhomogeneous spring-mass systems.

\section{Preliminaries}

Let $\mathbb{Z}, \mathbb{N}$ and $\mathbb{R}$ be the sets of all integers, natural and real numbers, respectively. Fix two real-valued sequences $\left(\theta_{i}\right),\left(\zeta_{i}\right), i \in \mathbb{Z}$, such that $\theta_{i}<\theta_{i+1}, \theta_{i} \leq \zeta_{i} \leq \theta_{i+1}$ for all $i \in \mathbb{Z},\left|\theta_{i}\right| \rightarrow \infty$ as $|i| \rightarrow \infty$, and assume that there exists a number $\theta>0$ such that $\theta_{i+1}-\theta_{i} \leq \theta, i \in \mathbb{Z}$.

The most general form of differential equations with piecewise constant argument of generalized type can be expressed as follows [3]

$$
x^{\prime}(t)=f(t, x(t), x(\gamma(t))),
$$

where $t \in \mathbb{R}, x \in \mathbb{R}^{n}, \gamma(t)=\zeta_{i}$ for $t \in\left[\theta_{i}, \theta_{i+1}\right), i \in \mathbb{Z}$. It is clear that the greatest integer function $[t]$ is a special case of the argument function $\gamma(t)$ of alternate type: retarded and advanced. In fact, $\gamma(t)=[t]$ for $\theta_{i}=\zeta_{i}=i, i \in \mathbb{Z}$.

In the present paper, we shall consider the following damped spring-mass systems with piecewise constant argument of generalized type

$$
m x^{\prime \prime}+c x^{\prime}+k x=A x(\gamma(t)),
$$

and

$$
m x^{\prime \prime}+c x^{\prime}+k x=A x(\gamma(t))+g(t),
$$

where $x \in \mathbb{R}, t \in \mathbb{R}, g(t)$ is a scalar, continuous real valued function, and $\gamma(t)=\zeta_{i}$, if $t \in\left[\theta_{i}, \theta_{i+1}\right), i \in \mathbb{Z}$. The systems (2.1) and (2.2) have discontinuities at the moments $\theta_{i}, i \in \mathbb{Z}$, since the piecewise function $\gamma(t)$ is not continuous. But the solutions of the systems (2.1) and (2.2) present a continuous and continuously differentiable dynamic motion within intervals $t \in\left[\theta_{i}, \theta_{i+1}\right), i \in \mathbb{Z}$.

Let $z_{1}=x$ and $z_{2}=x^{\prime}$. Then, spring-mass systems (2.1) and (2.2) can be reduced to the following first order differential equations, respectively,

$$
z^{\prime}(t)=A_{0} z(t)+A_{1} z(\gamma(t))
$$

and

$$
z^{\prime}(t)=A_{0} z(t)+A_{1} z(\gamma(t))+f(t) .
$$

Here the matrices $A_{0}=\left[\begin{array}{cc}0 & 1 \\ -\frac{k}{m} & -\frac{c}{m}\end{array}\right]$ and $A_{1}=\left[\begin{array}{cc}0 & 0 \\ \frac{A}{m} & 0\end{array}\right]$ depend on the parameters of the spring-mass systems (2.1) and (2.2). Moreover, $f(t)=\left[\begin{array}{c}0 \\ g(t)\end{array}\right]$. The systems (2.3) and (2.4) are linear homogeneous and nonhomogeneous systems with argument-function $\gamma(t)$, respectively.

In this study, our purpose is to examine damped spring-mass systems with generalized piecewise constant forces without transforming them into discrete equations and we shall assume that the systems exhibit harmonic motion with under damped, i.e., $\Delta=\frac{c^{2}}{m^{2}}-4 \frac{k}{m}<0$. While doing this examination, we use Floquet theory for differential equations with piecewise constant argument of generalized type and obtain sufficient conditions required for the spring-mass systems (2.3) and (2.4) to have periodic solutions [3]. Furthermore, we determine under which conditions the solutions of the system (2.3) 
exhibit stable, asymptotically stable and unstable behaviour with the help of the Floquet multipliers. Later, we give a result about the situation expressing that the system (2.4) has a unique periodic solution corresponding to the initial value. Additionally, we give numerical simulations, which show that the spring-mass system (2.3) has asymptotically stable or unstable solutions and the spring-mass system (2.4) has a periodic solution corresponding to special values chosen for the sequences and the parameters.

2.1. Definition. [3] A continuous function $z(t)$ is a solution of $(2.3)((2.4))$ on $\mathbb{R}$ if:

(i) the derivative $z^{\prime}(t)$ exists at each point $t \in \mathbb{R}$ with the possible exception of the points $\theta_{i}, i \in \mathbb{Z}$, where one-sided derivatives exist;

(ii) the equation $(2.3)((2.4))$ is satisfied for $z(t)$ on each interval $\left(\theta_{i}, \theta_{i+1}\right), i \in \mathbb{Z}$, and it holds for the right derivative of $z(t)$ at the points $\theta_{i}, i \in \mathbb{Z}$.

\section{The matrix-function of the linear homogeneous system}

Let us consider the linear homogenous part of (2.3) and (2.4) without piecewise constant argument as follows

$$
x^{\prime}(t)=A_{0} x(t)=\left[\begin{array}{cc}
0 & 1 \\
-\frac{k}{m} & -\frac{c}{m}
\end{array}\right] x(t) .
$$

Let I be a $2 \times 2$ identity matrix. Thus, the state transition matrix $X(t, s), t, s \in \mathbb{R}$, such that $X(s, s)=\mathrm{I}$, of the system (3.1) is given by

$$
\begin{aligned}
X(t, s) & =\mathrm{e}^{A_{0}(t-s)} \\
& =\mathrm{e}^{-\alpha(t-s)}\left[\begin{array}{ll}
\cos (\beta(t-s))+\frac{\alpha}{\beta} \sin (\beta(t-s)) & \frac{1}{\beta} \sin (\beta(t-s)) \\
-\frac{k}{m \beta} \sin (\beta(t-s)) & \cos (\beta(t-s))-\frac{\alpha}{\beta} \sin (\beta(t-s))
\end{array}\right]
\end{aligned}
$$

where $\alpha=\frac{c}{2 m}, \beta=\sqrt{\frac{k}{m}-\frac{c^{2}}{4 m^{2}}}=\frac{\sqrt{-\Delta}}{2}$.

Consider the following matrix-function

$$
M_{i}(t)=X\left(t, \zeta_{i}\right)+\int_{\zeta_{i}}^{t} X(t, s) A_{1}(s) d s, i \in \mathbb{Z},
$$

defined in [6]. This matrix-function is important for the results obtained below. For our systems (2.3) and (2.4) under consideration, we find that

$$
M_{i}(t)=\mathrm{e}^{-\alpha\left(t-\zeta_{i}\right)}\left[\begin{array}{cc}
K_{i} & L_{i} \\
M_{i} & N_{i}
\end{array}\right]
$$

where indices $K_{i}, L_{i}, M_{i}, N_{i}$ are evaluated as

$$
\begin{aligned}
K_{i} & =\left(1-\frac{A}{k}\right)\left(\cos \left(\beta\left(t-\zeta_{i}\right)\right)+\frac{\alpha}{\beta} \sin \left(\beta\left(t-\zeta_{i}\right)\right)\right)+\frac{A \mathrm{e}^{\alpha\left(t-\zeta_{i}\right)}}{k}, \\
L_{i} & =\frac{1}{\beta} \sin \left(\beta\left(t-\zeta_{i}\right)\right), \\
M_{i} & =-\frac{k}{m \beta}\left(1-\frac{A}{k}\right) \sin \left(\beta\left(t-\zeta_{i}\right)\right)
\end{aligned}
$$

and

$$
N_{i}=\cos \left(\beta\left(t-\zeta_{i}\right)\right)-\frac{\alpha}{\beta} \sin \left(\beta\left(t-\zeta_{i}\right)\right) .
$$


We shall need the following assumptions listed below:

(A1) $\frac{A}{k}\left(1-\mathrm{e}^{\alpha\left(t-\zeta_{i}\right)}\left(\cos \left(\beta\left(t-\zeta_{i}\right)\right)-\frac{\alpha}{\beta} \sin \left(\beta\left(t-\zeta_{i}\right)\right)\right)\right) \neq 1, \forall t \in\left[\theta_{i}, \theta_{i+1}\right], i \in \mathbb{Z}$;

(A2) $g$ is $\omega$-periodic;

(A3) There exists a number $p \in \mathbb{N}$ such that $\theta_{i+p}=\theta_{i}+\omega, \zeta_{i+p}=\zeta_{i}+\omega$, for all $i \in \mathbb{Z}$.

(A1) implies that the matrix-function $M_{i}(t)$ is invertible. (A2) and (A3) guarantee that the systems (2.3) and (2.4) are periodic.

3.1. Theorem. [6] For every $\left(t_{0}, z_{0}\right) \in \mathbb{R} \times \mathbb{R}^{2}$ there exists a unique solution $z(t)=$ $z\left(t, t_{0}, z_{0}\right)$ of (2.3) in the sense of Definition 2.1. such that $z\left(t_{0}\right)=z_{0}$ if and only if condition $(A 1)$ is valid.

3.2. Theorem. [6] A number $t_{0} \in \mathbb{R}, \theta_{i} \leq t_{0}<\theta_{i+1}$, is fixed. For every $z_{0} \in \mathbb{R}^{2}$ there exists a unique solution $z(t)=z\left(t, t_{0}, z_{0}\right)$ of (2.3) in the sense of Definition 2.1. such that $z\left(t_{0}\right)=z_{0}$ if and only if $\operatorname{det}\left[M_{i}\left(t_{0}\right)\right] \neq 0$ and $\operatorname{det}\left[M_{j}(t)\right] \neq 0$ for $t=\theta_{j}, \theta_{j+1}, j \in \mathbb{Z}$.

\section{The fundamental matrix of the homogeneous spring-mass sys- tem (2.3)}

Let us fix $t_{0} \in \mathbb{R}$. Then we can find an $i \in \mathbb{Z}$ such that $\theta_{i} \leq t_{0}<\theta_{i+1}$. Then there exists a fundamental matrix $Z(t)=Z\left(t, t_{0}\right)$ satisfying $Z\left(t_{0}\right)=\bar{Z}\left(t_{0}, t_{0}\right)=\mathrm{I}$ of the springmass system $(2.3)[3,5]$. Without loss of generality assume that $\theta_{i}<t_{0}<\zeta_{i}, i \in \mathbb{Z}$. For interval $t \in\left[t_{0}, \theta_{i+1}\right]$ which includes the initial point $t_{0}$, the fundamental matrix is

$$
Z\left(t, t_{0}\right)=Z(t)=M_{i}(t) M_{i}^{-1}\left(t_{0}\right) .
$$

Moreover, the fundamental matrix $Z(t)=Z\left(t, t_{0}\right)$ of $(2.3)$ is constructed in two different ways for increasing and decreasing $t$ as explained below [3, 5].

If $\theta_{i} \leq t_{0}<\theta_{i+1}, t \in\left[\theta_{l}, \theta_{l+1}\right], l>i$, then

$$
Z(t)=M_{l}(t)\left[\prod_{k=l}^{i+1} M_{k}^{-1}\left(\theta_{k}\right) M_{k-1}\left(\theta_{k}\right)\right] M_{i}^{-1}\left(t_{0}\right) .
$$

If $\theta_{i} \leq t_{0} \leq \theta_{i+1}, t \in\left[\theta_{j}, \theta_{j+1}\right], j<i$, then

$$
Z(t)=M_{j}(t)\left[\prod_{k=j}^{i-1} M_{k}^{-1}\left(\theta_{k+1}\right) M_{k+1}\left(\theta_{k+1}\right)\right] M_{i}^{-1}\left(t_{0}\right) .
$$

It can be shown that $Z(t, s)=Z(t) Z^{-1}(s), t, s \in \mathbb{R}$, and a solution $z(t), z\left(t_{0}\right)=z_{0}$, $\left(t_{0}, z_{0}\right) \in \mathbb{R} \times \mathbb{R}^{2}$, of $(2.3)$ is equal to $z(t)=Z\left(t, t_{0}\right) z_{0}, t \in \mathbb{R}$.

It's difficult to give a direct formula for the fundamental matrix of the spring-mass system (2.3). For illustration, we evaluate this matrix for the intervals $t \in\left[\theta_{i}, \theta_{i+1}\right]$, $t \in\left[\theta_{i+1}, \theta_{i+2}\right]$ where $t$ is increasing and for the interval $t \in\left[\theta_{i-1}, \theta_{i}\right]$ where $t$ is decreasing.

First, consider the interval $\left[\theta_{i}, \theta_{i+1}\right]$ which includes the initial value $t_{0}, \theta_{i} \leq \zeta_{i} \leq \theta_{i+1}$. Then, for $t \in\left[t_{0}, \theta_{i+1}\right]$ fundamental matrix of (2.3) has the form

$$
Z(t)=M_{i}(t) M_{i}^{-1}\left(t_{0}\right)=\frac{\mathrm{e}^{-\alpha\left(t-t_{0}\right)}}{s_{i_{1}}}\left[\begin{array}{cc}
K_{i_{1}} & L_{i_{1}} \\
M_{i_{1}} & N_{i_{1}}
\end{array}\right],
$$

and its indices are as follows

$$
\begin{aligned}
K_{i_{1}} & =\left(1-\frac{A}{k}\right)\left(\cos \left(\beta\left(t-t_{0}\right)\right)+\frac{\alpha}{\beta} \sin \left(\beta\left(t-t_{0}\right)\right)\right) \\
& +\frac{A \mathrm{e}^{\alpha\left(t-\zeta_{i}\right)}}{k}\left(\cos \left(\beta\left(t_{0}-\zeta_{i}\right)\right)-\frac{\alpha}{\beta} \sin \left(\beta\left(t_{0}-\zeta_{i}\right)\right)\right),
\end{aligned}
$$




$$
\begin{aligned}
L_{i_{1}} & =\frac{1}{\beta}\left(1-\frac{A}{k}\right) \sin \left(\beta\left(t-t_{0}\right)\right) \\
& +\frac{1}{\beta} \frac{A \mathrm{e}^{-\alpha \zeta_{i}}}{k}\left(\mathrm{e}^{\alpha t_{0}} \sin \left(\beta\left(t-\zeta_{i}\right)\right)-\mathrm{e}^{\alpha t} \sin \left(\beta\left(t_{0}-\zeta_{i}\right)\right)\right), \\
M_{i_{1}} & =-\frac{k}{m \beta}\left(1-\frac{A}{k}\right) \sin \left(\beta\left(t-t_{0}\right)\right), \\
N_{i_{1}} & =\left(1-\frac{A}{k}\right)\left(\cos \left(\beta\left(t-t_{0}\right)\right)-\frac{\alpha}{\beta} \sin \left(\beta\left(t-t_{0}\right)\right)\right) \\
& +\frac{A \mathrm{e}^{\alpha\left(t_{0}-\zeta_{i}\right)}}{k}\left(\cos \left(\beta\left(t-\zeta_{i}\right)\right)-\frac{\alpha}{\beta} \sin \left(\beta\left(t-\zeta_{i}\right)\right)\right), \\
s_{i_{1}} & =1-\frac{A}{k}\left(1-\mathrm{e}^{\alpha\left(t_{0}-\zeta_{i}\right)}\left(\cos \left(\beta\left(t_{0}-\zeta_{i}\right)\right)-\frac{\alpha}{\beta} \sin \left(\beta\left(t_{0}-\zeta_{i}\right)\right)\right)\right) .
\end{aligned}
$$

Second, for $t \in\left[\theta_{i-1}, \theta_{i}\right], \theta_{i-1} \leq \zeta_{i-1} \leq \theta_{i}, i \in \mathbb{Z}$, we find the fundamental matrix of (2.3) as follows

$$
Z(t)=M_{i-1}(t) M_{i-1}^{-1}\left(\theta_{i}\right) M_{i}\left(\theta_{i}\right) M_{i}^{-1}\left(t_{0}\right)=\frac{\mathrm{e}^{-\alpha\left(t-t_{0}\right)}}{s_{i_{1}} s_{i_{2}}}\left[\begin{array}{cc}
K_{i_{2}} & L_{i_{2}} \\
M_{i_{2}} & N_{i_{2}}
\end{array}\right],
$$

where

$$
\begin{aligned}
& K_{i_{2}}=\left(1-\frac{A}{k}\right)^{2}\left(\cos \left(\beta\left(t-t_{0}\right)\right)+\frac{\alpha}{\beta} \sin \left(\beta\left(t-t_{0}\right)\right)\right) \\
& +\frac{A}{k}\left(1-\frac{A}{k}\right)\left(\mathrm{e}^{\alpha\left(t-\zeta_{i-1}\right)}\left(\cos \left(\beta\left(\zeta_{i-1}-t_{0}\right)\right)+\frac{\alpha}{\beta} \sin \left(\beta\left(\zeta_{i-1}-t_{0}\right)\right)\right)\right. \\
& +\mathrm{e}^{\alpha\left(\theta_{i}-\zeta_{i}\right)}\left(\cos \left(\beta\left(t-t_{0}-\theta_{i}+\zeta_{i}\right)\right)+\frac{\alpha}{\beta} \sin \left(\beta\left(t-t_{0}-\theta_{i}+\zeta_{i}\right)\right)\right. \\
& \left.-\left(\frac{k}{m \beta^{2}}\right) \sin \left(\beta\left(t-\theta_{i}\right)\right) \sin \left(\beta\left(t_{0}-\zeta_{i}\right)\right)\right) \\
& +\frac{A}{m \beta^{2}}\left(1-\frac{A}{k}\right) \mathrm{e}^{\alpha\left(\theta_{i}-\zeta_{i-1}\right)} \sin \left(\beta\left(t-\zeta_{i-1}\right)\right) \sin \left(\beta\left(t_{0}-\theta_{i}\right)\right) \\
& +\frac{A^{2}}{k^{2}} \mathrm{e}^{\alpha\left(t-\zeta_{i}+\theta_{i}-\zeta_{i-1}\right)}\left(\cos \left(\beta\left(t_{0}+\theta_{i}-\zeta_{i}-\zeta_{i-1}\right)\right)-\frac{\alpha}{\beta} \sin \left(\beta\left(t_{0}+\theta_{i}-\zeta_{i}-\zeta_{i-1}\right)\right)\right. \\
& \left.+\frac{k}{m \beta^{2}} \sin \left(\beta\left(\theta_{i}-\zeta_{i-1}\right)\right) \sin \left(\beta\left(t_{0}-\zeta_{i}\right)\right)\right) \text {, } \\
& L_{i_{2}}=\frac{1}{\beta}\left(1-\frac{A}{k}\right)^{2} \sin \left(\beta\left(t-t_{0}\right)\right) \\
& +\frac{A}{k \beta}\left(1-\frac{A}{k}\right)\left(\mathrm{e}^{\alpha\left(t-\zeta_{i-1}\right)} \sin \left(\beta\left(\zeta_{i-1}-t_{0}\right)\right)+\mathrm{e}^{\alpha\left(t_{0}-\zeta_{i}\right)} \sin \left(\beta\left(t-\zeta_{i}\right)\right)\right. \\
& -\mathrm{e}^{\alpha\left(\theta_{i}-\zeta_{i}\right)} \sin \left(\beta\left(t_{0}-\zeta_{i}\right)\right)\left(\cos \left(\beta\left(t-\theta_{i}\right)\right)+\frac{\alpha}{\beta} \sin \left(\beta\left(t-\theta_{i}\right)\right)\right) \\
& \left.+\mathrm{e}^{\alpha\left(\theta_{i}-\zeta_{i-1}\right)} \sin \left(\beta\left(t-\zeta_{i-1}\right)\right)\left(\cos \left(\beta\left(t_{0}-\theta_{i}\right)\right)+\frac{\alpha}{\beta} \sin \left(\beta\left(t_{0}-\theta_{i}\right)\right)\right)\right) \\
& +\frac{A^{2}}{k^{2} \beta}\left(-\mathrm{e}^{\alpha\left(t-\zeta_{i-1}+\theta_{i}-\zeta_{i}\right)} \sin \left(\beta\left(t_{0}-\zeta_{i}\right)\right)\left(\cos \left(\beta\left(\theta_{i}-\zeta_{i-1}\right)\right)-\frac{\alpha}{\beta} \sin \left(\beta\left(\theta_{i}-\zeta_{i-1}\right)\right)\right)\right. \\
& +\mathrm{e}^{\alpha\left(t-\zeta_{i-1}+t_{0}-\zeta_{i}\right)} \sin \left(\beta\left(\zeta_{i-1}-\zeta_{i}\right)\right) \\
& \left.+\mathrm{e}^{\alpha\left(t_{0}-\zeta_{i-1}+\theta_{i}-\zeta_{i}\right)} \sin \left(\beta\left(t-\zeta_{i-1}\right)\right)\left(\cos \left(\beta\left(\theta_{i}-\zeta_{i}\right)\right)-\frac{\alpha}{\beta} \sin \left(\beta\left(\theta_{i}-\zeta_{i}\right)\right)\right)\right),
\end{aligned}
$$




$$
\begin{aligned}
& M_{i_{2}}=-\frac{k}{m \beta}\left(1-\frac{A}{k}\right)^{2} \sin \left(\beta\left(t-t_{0}\right)\right) \\
& +\frac{A}{m \beta}\left(1-\frac{A}{k}\right)\left(-\mathrm{e}^{\alpha\left(\theta_{i}-\zeta_{i}\right)} \sin \left(\beta\left(t-\theta_{i}\right)\right)\left(\cos \left(\beta\left(t_{0}-\zeta_{i}\right)\right)-\frac{\alpha}{\beta} \sin \left(\beta\left(t_{0}-\zeta_{i}\right)\right)\right)\right. \\
& -\mathrm{e}^{\alpha\left(\theta_{i}-\zeta_{i-1}\right)} \sin \left(\beta\left(\theta_{i}-\zeta_{i}\right)\right)\left(\cos \left(\beta\left(t-\zeta_{i-1}+t_{0}-\zeta_{i}\right)\right)-\frac{\alpha}{\beta} \sin \left(\beta\left(t-\zeta_{i-1}+t_{0}-\zeta_{i}\right)\right)\right) \\
& +\mathrm{e}^{\alpha\left(\theta_{i}-\zeta_{i-1}\right)} \sin \left(\beta\left(t_{0}-\zeta_{i}\right)\right)\left(\cos \left(\beta\left(t-\zeta_{i-1}+\theta_{i}-\zeta_{i}\right)\right)-\frac{\alpha}{\beta} \sin \left(\beta\left(t-\zeta_{i-1}+\theta_{i}-\zeta_{i}\right)\right)\right), \\
& N_{i_{2}}=\left(1-\frac{A}{k}\right)^{2}\left(\cos \left(\beta\left(t-t_{0}\right)\right)-\frac{\alpha}{\beta} \sin \left(\beta\left(t-t_{0}\right)\right)\right) \\
& +\frac{A}{k}\left(1-\frac{A}{k}\right)\left(\mathrm{e}^{\alpha\left(t_{0}-\zeta_{i}\right)}\left(\cos \left(\beta\left(t-\zeta_{i}\right)\right)-\frac{\alpha}{\beta} \sin \left(\beta\left(t-\zeta_{i}\right)\right)\right)\right. \\
& +\left(\mathrm{e}^{\alpha\left(\theta_{i}-\zeta_{i}\right)} \sin \left(\beta\left(t_{0}-\zeta_{i}\right)\right) \sin \left(\beta\left(t-\theta_{i}\right)\right) \frac{k}{m \beta^{2}}\right) \\
& +\mathrm{e}^{\alpha\left(\theta_{i}-\zeta_{i-1}\right)}\left(\cos \left(\beta\left(t-t_{0}+\theta_{i}-\zeta_{i-1}\right)\right)-\frac{\alpha}{\beta} \sin \left(\beta\left(t-t_{0}+\theta_{i}-\zeta_{i-1}\right)\right)\right. \\
& \left.-\mathrm{e}^{\alpha\left(\theta_{i}-\zeta_{i-1}\right)} \sin \left(\beta\left(t-\zeta_{i-1}\right)\right) \cos \left(\beta\left(t_{0}-\theta_{i}\right)\right) \frac{k}{m \beta^{2}}\right) \\
& +\frac{A^{2}}{k^{2}} \mathrm{e}^{\alpha\left(\theta_{i}-\zeta_{i-1}+t_{0}-\zeta_{i}\right)}\left(\cos \left(\beta\left(t-\zeta_{i-1}+\theta_{i}-\zeta_{i}\right)\right)-\frac{\alpha}{\beta} \sin \left(\beta\left(t-\zeta_{i-1}+\theta_{i}-\zeta_{i}\right)\right)\right. \\
& \left.+\sin \left(\beta\left(t-\zeta_{i-1}\right)\right) \sin \left(\beta\left(\theta_{i}-\zeta_{i}\right)\right) \frac{k}{m \beta^{2}}\right), \\
& s_{i_{1}}=1-\frac{A}{k}\left(1-\mathrm{e}^{\alpha\left(t_{0}-\zeta_{i}\right)}\left(\cos \left(\beta\left(t_{0}-\zeta_{i}\right)\right)-\frac{\alpha}{\beta} \sin \left(\beta\left(t_{0}-\zeta_{i}\right)\right)\right)\right), \\
& s_{i_{2}}=1-\frac{A}{k}\left(1-\mathrm{e}^{\alpha\left(\theta_{i}-\zeta_{i-1}\right)}\left(\cos \left(\beta\left(\theta_{i}-\zeta_{i-1}\right)\right)-\frac{\alpha}{\beta} \sin \left(\beta\left(\theta_{i}-\zeta_{i-1}\right)\right)\right)\right) .
\end{aligned}
$$

Finally, for $t \in\left[\theta_{i+1}, \theta_{i+2}\right], \theta_{i+1} \leq \zeta_{i+1} \leq \theta_{i+2}, i \in \mathbb{Z}$, where $t$ is increasing, fundamental matrix of (2.3) is

$$
Z(t)=M_{i+1}(t) M_{i+1}^{-1}\left(\theta_{i+1}\right) M_{i}\left(\theta_{i+1}\right) M_{i}^{-1}\left(t_{0}\right)=\frac{\mathrm{e}^{-\alpha\left(t-t_{0}\right)}}{s_{i_{1}} s_{i_{3}}}\left[\begin{array}{cc}
K_{i_{3}} & L_{i_{3}} \\
M_{i_{3}} & N_{i_{3}}
\end{array}\right],
$$

where the indices are given by

$$
\begin{aligned}
K_{i_{3}} & =\left(1-\frac{A}{k}\right)^{2}\left(\cos \left(\beta\left(t-t_{0}\right)\right)+\frac{\alpha}{\beta} \sin \left(\beta\left(t-t_{0}\right)\right)\right) \\
& +\frac{A}{k}\left(1-\frac{A}{k}\right)\left(\mathrm{e}^{\alpha\left(t-\zeta_{i+1}\right)}\left(\cos \left(\beta\left(t_{0}-\zeta_{i+1}\right)\right)-\frac{\alpha}{\beta} \sin \left(\beta\left(t_{0}-\zeta_{i+1}\right)\right)\right)\right. \\
& +\mathrm{e}^{\alpha\left(\theta_{i+1}-\zeta_{i}\right)}\left(\cos \left(\beta\left(t-t_{0}-\theta_{i+1}+\zeta_{i}\right)\right)\right. \\
& \left.\left.+\frac{\alpha}{\beta} \sin \left(\beta\left(t-t_{0}-\theta_{i+1}+\zeta_{i}\right)\right)-\frac{k}{m \beta^{2}} \sin \left(\beta\left(t-\theta_{i+1}\right)\right) \sin \left(\beta\left(t_{0}-\zeta_{i}\right)\right)\right)\right) \\
& +\frac{A}{m \beta^{2}}\left(1-\frac{A}{k}\right) \mathrm{e}^{\alpha\left(\theta_{i+1}-\zeta_{i+1}\right)} \sin \left(\beta\left(t-\zeta_{i+1}\right)\right) \sin \left(\beta\left(t_{0}-\theta_{i+1}\right)\right) \\
& +\frac{A^{2}}{k^{2}} \mathrm{e}^{\alpha\left(t-\zeta_{i}+\theta_{i+1}-\zeta_{i+1}\right)}\left(\cos \left(\beta\left(t_{0}+\theta_{i+1}-\zeta_{i}-\zeta_{i+1}\right)\right)\right. \\
& \left.-\frac{\alpha}{\beta} \sin \left(\beta\left(t_{0}+\theta_{i+1}-\zeta_{i}-\zeta_{i+1}\right)\right)+\sin \left(\beta\left(\theta_{i+1}-\zeta_{i+1}\right)\right) \sin \left(\beta\left(t_{0}-\zeta_{i}\right)\right) \frac{k}{m \beta^{2}}\right),
\end{aligned}
$$




$$
\begin{aligned}
& L_{i_{3}}=\frac{1}{\beta}\left(1-\frac{A}{k}\right)^{2} \sin \left(\beta\left(t-t_{0}\right)\right) \\
& +\frac{A}{k \beta}\left(1-\frac{A}{k}\right)\left(-\mathrm{e}^{\alpha\left(t-\zeta_{i+1}\right)} \sin \left(\beta\left(t_{0}-\zeta_{i+1}\right)\right)\right. \\
& +\mathrm{e}^{\alpha\left(\theta_{i+1}-\zeta_{i+1}\right)} \sin \left(\beta\left(t-\zeta_{i+1}\right)\right)\left(\cos \left(\beta\left(t_{0}-\theta_{i+1}\right)\right)+\frac{\alpha}{\beta} \sin \left(\beta\left(t_{0}-\theta_{i+1}\right)\right)\right) \\
& -\mathrm{e}^{\alpha\left(\theta_{i+1}-\zeta_{i}\right)} \sin \left(\beta\left(t_{0}-\zeta_{i}\right)\right)\left(\cos \left(\beta\left(t-\theta_{i+1}\right)\right)+\frac{\alpha}{\beta} \sin \left(\beta\left(t-\theta_{i+1}\right)\right)\right) \\
& \left.+\mathrm{e}^{\alpha\left(t_{0}-\zeta_{i}\right)} \sin \left(\beta\left(t-\zeta_{i}\right)\right)\right) \\
& +\frac{A^{2}}{k^{2} \beta}\left(\mathrm{e}^{\alpha\left(t_{0}-\zeta_{i+1}+\theta_{i+1}-\zeta_{i}\right)} \sin \left(\beta\left(t-\zeta_{i+1}\right)\right)\left(\cos \left(\beta\left(\theta_{i+1}-\zeta_{i}\right)\right)-\frac{\alpha}{\beta} \sin \left(\beta\left(\theta_{i+1}-\zeta_{i}\right)\right)\right)\right. \\
& -\mathrm{e}^{\alpha\left(t-\zeta_{i+1}+t_{0}-\zeta_{i}\right)} \sin \left(\beta\left(\zeta_{i}-\zeta_{i+1}\right)\right) \\
& \left.-\mathrm{e}^{\alpha\left(t-\zeta_{i+1}+\theta_{i+1}-\zeta_{i}\right)} \sin \left(\beta\left(t_{0}-\zeta_{i}\right)\right)\left(\cos \left(\beta\left(\theta_{i+1}-\zeta_{i+1}\right)\right)-\frac{\alpha}{\beta} \sin \left(\beta\left(\theta_{i+1}-\zeta_{i+1}\right)\right)\right)\right), \\
& M_{i_{3}}=-\frac{k}{m \beta}\left(1-\frac{A}{k}\right) \sin \left(\beta\left(t-t_{0}\right)\right) \\
& +\frac{A}{m \beta}\left(1-\frac{A}{k}\right)\left(-\mathrm{e}^{\alpha\left(\theta_{i+1}-\zeta_{i}\right)} \sin \left(\beta\left(t-\theta_{i+1}\right)\right)\left(\cos \left(\beta\left(t_{0}-\zeta_{i}\right)\right)-\frac{\alpha}{\beta} \sin \left(\beta\left(t_{0}-\zeta_{i}\right)\right)\right)\right. \\
& \left.+\mathrm{e}^{\alpha\left(\theta_{i+1}-\zeta_{i+1}\right)} \sin \left(\beta\left(t_{0}-\theta_{i+1}\right)\right)\left(\cos \left(\beta\left(t-\zeta_{i+1}\right)\right)-\frac{\alpha}{\beta} \sin \left(\beta\left(t-\zeta_{i+1}\right)\right)\right)\right), \\
& N_{i_{3}}=\left(1-\frac{A}{k}\right)^{2}\left(\cos \left(\beta\left(t-t_{0}\right)\right)-\frac{\alpha}{\beta} \sin \left(\beta\left(t-t_{0}\right)\right)\right) \\
& +\frac{A}{k}\left(1-\frac{A}{k}\right)\left(\mathrm{e}^{\alpha\left(t_{0}-\zeta_{i}\right)}\left(\cos \left(\beta\left(t-\zeta_{i}\right)\right)-\frac{\alpha}{\beta} \sin \left(\beta\left(t-\zeta_{i}\right)\right)\right)\right. \\
& +\left(\mathrm{e}^{\alpha\left(\theta_{i+1}-\zeta_{i}\right)} \sin \left(\beta\left(t_{0}-\zeta_{i}\right)\right) \sin \left(\beta\left(t-\theta_{i+1}\right)\right) \frac{k}{m \beta^{2}}\right) \\
& +\mathrm{e}^{\alpha\left(\theta_{i+1}-\zeta_{i+1}\right)}\left(\cos \left(\beta\left(t-t_{0}+\theta_{i+1}-\zeta_{i+1}\right)\right)-\frac{\alpha}{\beta} \sin \left(\beta\left(t-t_{0}+\theta_{i+1}-\zeta_{i+1}\right)\right)\right. \\
& -\left(\sin \left(\beta\left(t-\zeta_{i+1}\right)\right) \sin \left(\beta\left(t_{0}-\theta_{i+1}\right)\right) \frac{k}{m \beta^{2}}\right) \\
& +\frac{A^{2}}{k^{2}} \mathrm{e}^{\alpha\left(\theta_{i+1}-\zeta_{i+1}+t_{0}-\zeta_{i}\right)}\left(\cos \left(\beta\left(t-\zeta_{i+1}+\theta_{i+1}-\zeta_{i}\right)\right)-\frac{\alpha}{\beta} \sin \left(\beta\left(t-\zeta_{i+1}+\theta_{i+1}-\zeta_{i}\right)\right)\right. \\
& \left.+\sin \left(\beta\left(t-\zeta_{i+1}\right)\right) \sin \left(\beta\left(\theta_{i+1}-\zeta_{i}\right)\right) \frac{k}{m \beta^{2}}\right), \\
& s_{i_{1}}=1-\frac{A}{k}\left(1-\mathrm{e}^{\alpha\left(t_{0}-\zeta_{i}\right)}\left(\cos \left(\beta\left(t_{0}-\zeta_{i}\right)\right)-\frac{\alpha}{\beta} \sin \left(\beta\left(t_{0}-\zeta_{i}\right)\right)\right)\right)
\end{aligned}
$$

and

$s_{i_{3}}=1-\frac{A}{k}\left(1-\mathrm{e}^{\alpha\left(\theta_{i+1}-\zeta_{i+1}\right)}\left(\cos \left(\beta\left(\theta_{i+1}-\zeta_{i+1}\right)\right)-\frac{\alpha}{\beta} \sin \left(\beta\left(\theta_{i+1}-\zeta_{i+1}\right)\right)\right)\right)$.

Clearly, it is not easy to obtain a complete form for the fundamental matrix. So here, we have created the fundamental matrix of the system (2.3) in three intervals for illustration.

The following theorem about existence and uniqueness of solutions for the spring-mass system (2.4) can be given. 
4.1. Theorem. [3] Assume that the condition $(A 1)$ is fulfilled. Then for every $\left(t_{0}, z_{0}\right) \in$ $\mathbb{R} \times \mathbb{R}^{2}$ there exists a unique solution $z(t)=z\left(t, t_{0}, z_{0}\right)$ of (2.4) in the sense of Definition 2.1. such that $z\left(t_{0}\right)=z_{0}$, and

$$
z(t)=Z\left(t, t_{0}\right) z_{0}+\int_{t_{0}}^{t} Z(t, s) f(s) d s
$$

\section{Main results}

Let $t_{0}=0$ and denote by $Z(\omega)=Z\left(\omega, t_{0}\right)=Z(\omega, 0)$ the matrix of monodromy. The eigenvalues $\rho$ of the monodromy matrix $Z(\omega)$ are called Floquet multipliers (or simply multipliers) of system (2.3). The eigenvalues of the matrix $P=\frac{1}{\omega} \ln Z(\omega), \lambda$, are called the Floquet exponents (or simply exponents) [3].

In this section, for the $\omega$-periodic spring-mass systems (2.3) and (2.4), we shall state the results depending on the multipliers $\rho_{1}$ and $\rho_{2}$. $\omega$ can be located in one of the intervals $\left[\theta_{i}, \theta_{i+1}\right]$ which includes $t_{0},\left[\theta_{l}, \theta_{l+1}\right], l>i$ where $t$ is increasing or $\left[\theta_{j}, \theta_{j+1}\right], j<i$ where $t$ is decreasing. The monodromy matrix can be found using the constructions presented in the Section 4. Thus, the periodicity of the solutions for the systems (2.3), (2.4) and stability of the solutions for the system (2.3) can be investigated using the multipliers. These results about periodicity and stability are stated by the following theorems [3].

5.1. Theorem. If $\rho$ is a multiplier then there exists a nontrivial solution $z(t)$ of (2.3) such that $z(t+\omega)=\rho z(t)$. Conversely, if there exists a nontrivial solution $z(t)$ of (2.3) such that $z(t+\omega)=\rho z(t)$, then $\rho$ is a multiplier.

5.2. Theorem. System (2.3) has a $\sigma \omega$-periodic solution if and only if there exists a multiplier $\rho$ such that $\rho^{\sigma}=1, \sigma \in \mathbb{Z}$.

We can say that the spring-mass system (2.4) has a unique $\omega$-periodic solution under the assumptions given in the next theorem.

5.3. Theorem. If unity is not one of the multipliers, then (2.4) has a unique $\omega$-periodic solution $z(t)$ such that

$$
z(0)=z_{0}=[I-Z(\omega)]^{-1} \int_{0}^{\omega} Z(\omega, s) f(s) d s .
$$

Moreover, for the solutions of (2.3), it is possible to analyze stability by Floquet multipliers as given below.

5.4. Theorem. The solutions of (2.3) are

(i) stable if $\left|\rho_{j}\right| \leq 1$ for $\forall j=1,2$ and $\rho_{j}$ is simple when $\left|\rho_{j}\right|=1$;

(ii) asymptotically stable if and only if $\left|\rho_{j}\right|<1, \forall j=1,2$;

(iii) unstable if there exists a multiplier for some $j=1,2$ such that $\left|\rho_{j}\right|>1$.

If, for example, $\omega$ belongs to one of the intervals $\left[\theta_{i}, \theta_{i+1}\right],\left[\theta_{i-1}, \theta_{i}\right]$ or $\left[\theta_{i+1}, \theta_{i+2}\right]$, $i \in \mathbb{Z}$, then the monodromy matrix and thus multipliers can be computed by using the equations (4.1), (4.2) and (4.3), respectively. It is possible to find the multipliers for other cases similarly.

\section{The numerical simulations}

In this section, we give examples with simulations to see more concrete results. Take the initial value $t_{0}=0$ for all of these examples. 
6.1. Example. First, let the parameters be $m=c=A=1, k=40$ and the sequences be $\theta_{i}=\zeta_{i}=\frac{1}{10} i$. In other words, consider the damped spring mass system

$$
x^{\prime \prime}+x^{\prime}+40 x=x(\gamma(t)) .
$$

Considering $z_{1}=x$ and $z_{2}=x^{\prime}$, spring-mass system (6.1) can be reduced to the following first order differential equation

$$
z^{\prime}(t)=\left[\begin{array}{cc}
0 & 1 \\
-40 & -1
\end{array}\right] z(t)+\left[\begin{array}{ll}
0 & 0 \\
1 & 0
\end{array}\right] z(\gamma(t))
$$

Since the conditions $(A 1)$ and $(A 3)$ are satisfied, the equation (6.1) and so the system (6.2) are 0.1 -periodic. $t=\omega=0.1 \in\left[\theta_{0}, \theta_{1}\right]=[0,0.1]$. Then, for $t \in[0,0.1]$, the monodromy matrix is in the following form

$$
Z(0.1)=\left[\begin{array}{ll}
0.8187863983 & 0.08894511882 \\
-3.468859634 & 0.7238801333
\end{array}\right]
$$

Thus, we find the multipliers $\rho_{1}=0.7713332658+0.5534314163 i$ and $\rho_{2}=0.7713332658-$ $0.5534314163 i$. So, $\left|\rho_{1}\right|=\left|\rho_{2}\right|=0.9493373159<1$. According to Theorem 5.1., there exist nontrivial solutions $z(t+0.1)=\rho_{1} z(t)$ and $z(t+0.1)=\rho_{2} z(t)$ of the system (6.2). It is seen that the solutions of (6.2) are asymptotically stable, in particular, the fixed point $(0,0)$ of the system (6.2) is asymptotically stable. The simulations showing asymptotic stability of the trivial solution of (6.2) are given in Figure 1. 

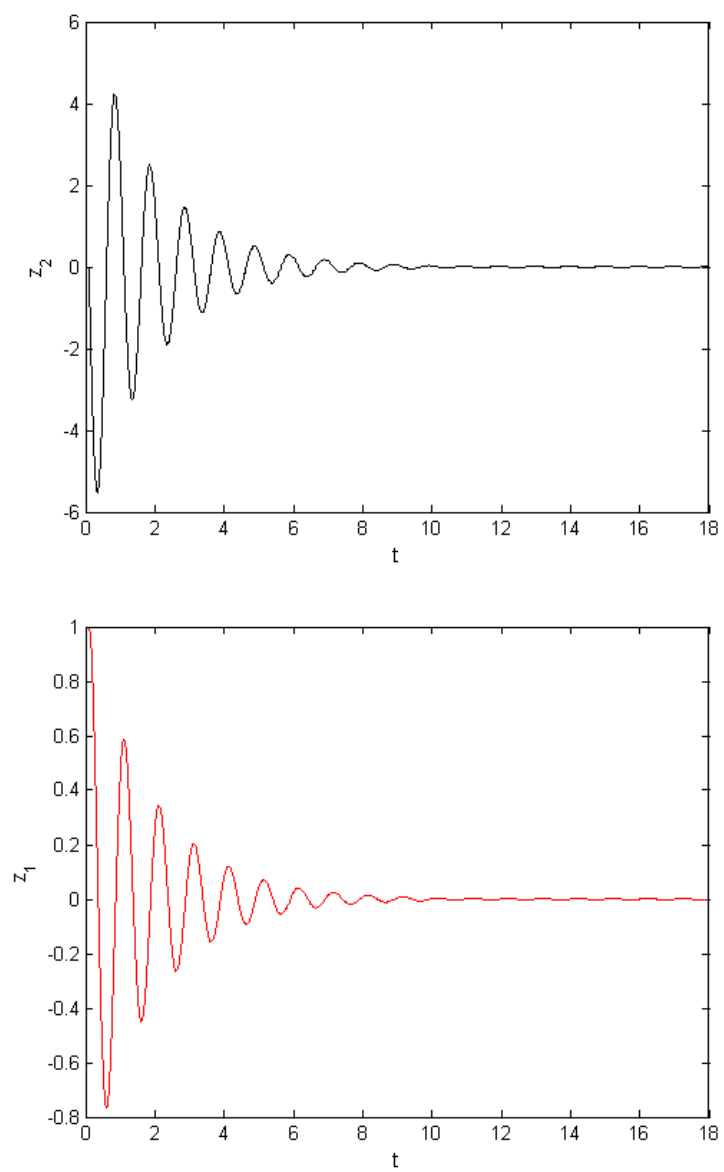

Figure 1. Simulations showing the asymptotic stability of the trivial solution of (6.2) for $t \in[0,18]$ with $m=c=A=1, k=40, \theta_{i}=\zeta_{i}=$ $\frac{1}{10} i, i \in \mathbb{Z}$ starting at $\left(z_{1}(0), z_{2}(0)\right)=(1,0)$

If we continue for more intervals, we obtain the simulations given in Figure 2.

6.2. Example. Second, let $m=1, c=0.00000002, k=25.0000000000001, A=1$ and the sequences be $\theta_{i}=\zeta_{i}=\frac{1}{10} i$. So, consider the spring mass system

$$
x^{\prime \prime}+2 \times 10^{-8} x^{\prime}+25 \times 10^{-13} x=x(\gamma(t)) .
$$

Considering $z_{1}=x$ and $z_{2}=x^{\prime}$, spring-mass system (6.3) can be reduced to the following first order differential equation

$$
z^{\prime}(t)=\left[\begin{array}{cc}
0 & 1 \\
-25 \times 10^{-13} & -2 \times 10^{-8}
\end{array}\right] z(t)+\left[\begin{array}{ll}
0 & 0 \\
1 & 0
\end{array}\right] z(\gamma(t)) .
$$

It can be shown that the conditions $(A 1)$ and $(A 3)$ are satisfied. Hence, the equation (6.3) and so the system (6.4) are 0.1-periodic. $t=\omega=0.1 \in\left[\theta_{0}, \theta_{1}\right]=[0,0.1]$. Then, 

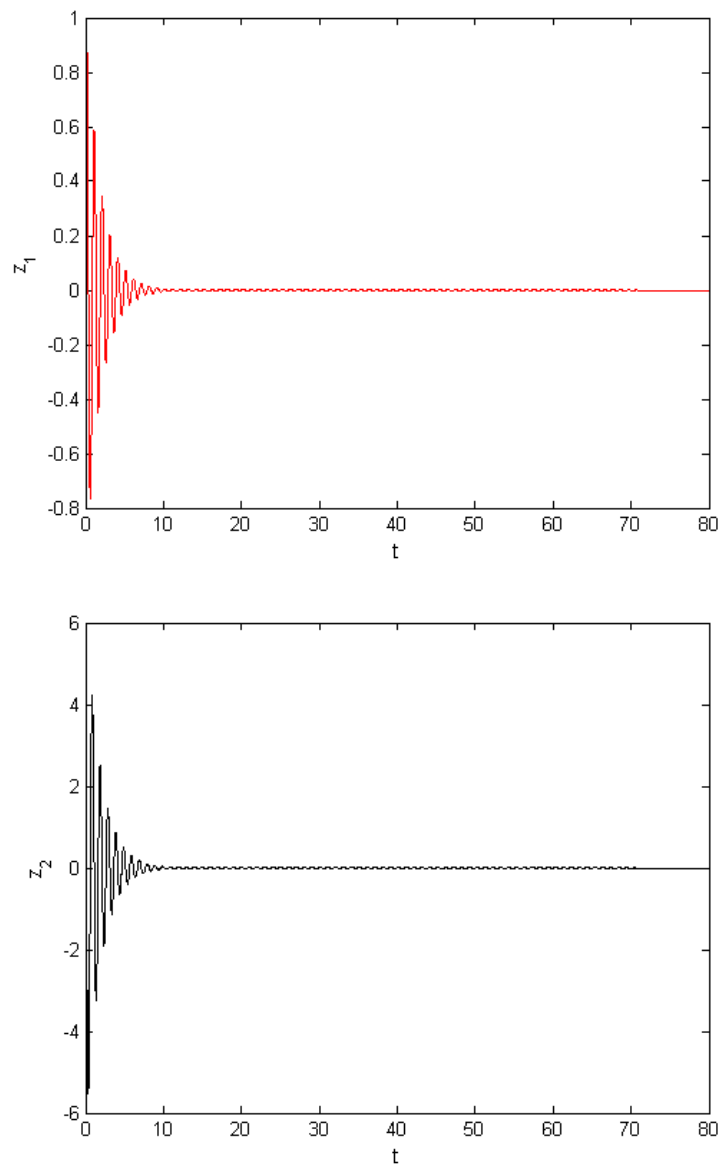

Figure 2. Simulations showing the asymptotic stability of the trivial solution of (6.2) for $t \in[0,80]$ with $m=c=A=1, k=40, \theta_{i}=\zeta_{i}=$ $\frac{1}{10} i, i \in \mathbb{Z}$ starting at $\left(z_{1}(0), z_{2}(0)\right)=(1,0)$

for $t \in[0,0.1]$, the monodromy matrix is

$$
Z(0.1)=\left[\begin{array}{ll}
0.8824792595 & 0.09588510762 \\
-2.301242583 & 0.8775825600
\end{array}\right]
$$

Thus, the multipliers are $\rho_{1}=0.88003091+0.4697327946 i$ and $\rho_{2}=0.88003091-$ $0.4697327946 i$. So, $\left|\rho_{1}\right|=\left|\rho_{2}\right|=0.9975486457<1$. Then Theorem 5.1. implies that there exist nontrivial solutions $z(t+0.1)=\rho_{1} z(t)$ and $z(t+0.1)=\rho_{2} z(t)$ of the system (6.4). It is seen that the solutions of (6.4) are asymptotically stable, in particular, the fixed point $(0,0)$ of the system $(6.4)$ is asymptotically stable. The simulations showing asymptotic stability of the trivial solution of (6.4) are given in Figure 3. 

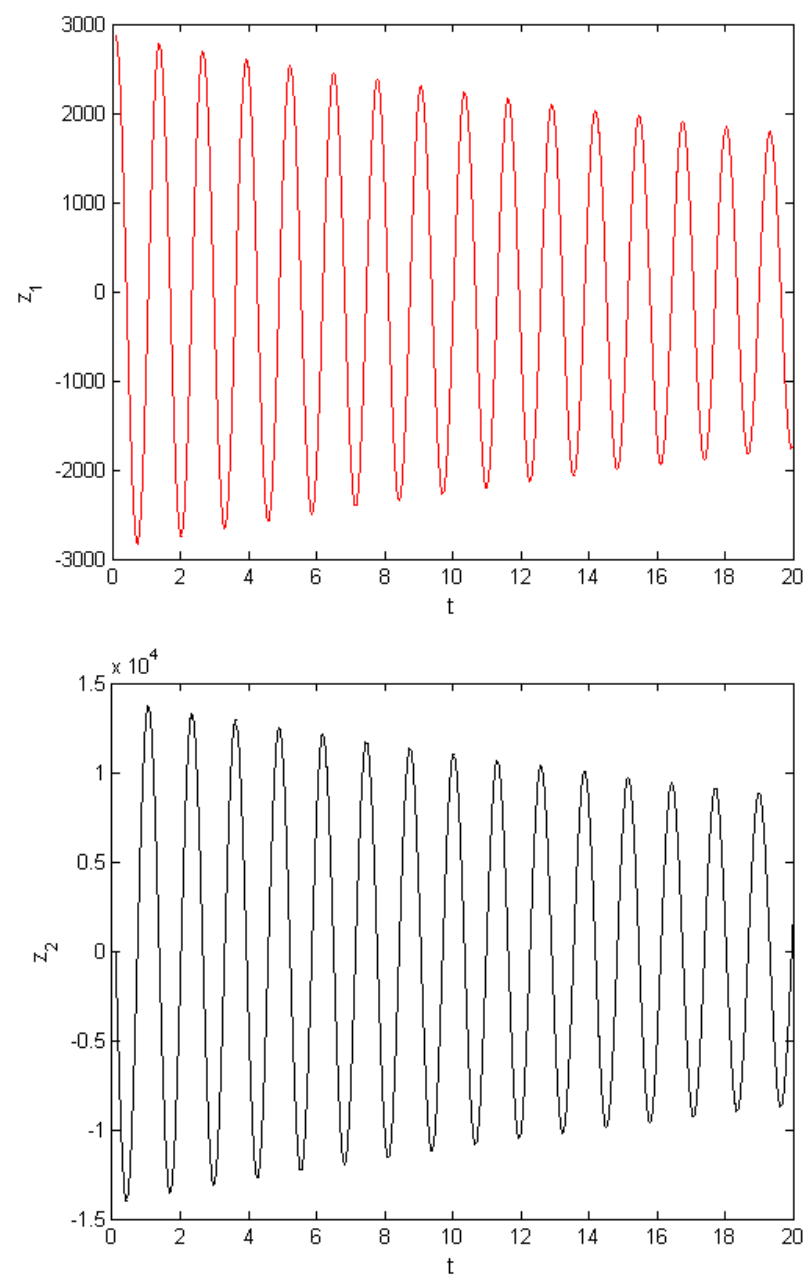

Figure 3. Simulations showing the asymptotic stability of the trivial solution of (6.4) for $t \in[0,20]$ with $m=1, c=0.00000002$, $k=25.0000000000001, A=1, \theta_{i}=\zeta_{i}=\frac{1}{10} i, i \in \mathbb{Z}$ starting at $\left(z_{1}(0), z_{2}(0)\right)=(2870.48457639202570,-0.0158952790662500004)$

If we continue for more intervals, we see that the solution $z_{1}(t)$ is asymptotically stable with the simulation in Figure 4. Similarly, for more intervals, it can be seen that the other solution is also asymptotically stable. 


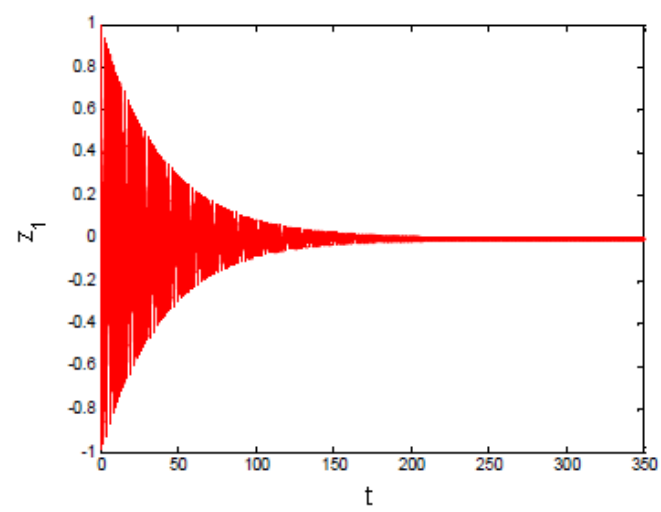

Figure 4. Simulation showing the asymptotic stability of the trivial solution of (6.4) for $t \in[0,350]$ with $m=1, c=0.00000002$, $k=25.0000000000001, A=1, \theta_{i}=\zeta_{i}=\frac{1}{10} i, i \in \mathbb{Z}$ starting at $\left(z_{1}(0), z_{2}(0)\right)=(2870.48457639202570,-0.0158952790662500004)$

6.3. Example. For $k=10, m=1, c=0.01, A=0.00000001$ and the sequences $\theta_{i}=\zeta_{i}=\frac{1}{10} i$, consider the linear nonhomogeneous spring-mass system with piecewise constant argument of generalized type

$$
x^{\prime \prime}+0.01 x^{\prime}+10 x=10^{-8} x(\gamma(t))+4 \cos (20 \pi t) .
$$

Taking $z_{1}=x$ and $z_{2}=x^{\prime}$, spring-mass system (6.5) can be reduced to the following nonhomogeneous differential equation

$$
z^{\prime}(t)=\left[\begin{array}{cc}
0 & 1 \\
-10 & -0.01
\end{array}\right] z(t)+\left[\begin{array}{cc}
0 & 0 \\
10^{-8} & 0
\end{array}\right] z(\gamma(t))+\left[\begin{array}{c}
0 \\
4 \cos (20 \pi t)
\end{array}\right]
$$

Since the conditions $(A 1)$ and $(A 3)$ are satisfied, the equation (6.5) and so the system (6.6) are 0.1 -periodic. $t=\omega=0.1 \in\left[\theta_{0}, \theta_{1}\right]=[0,0.1]$. Then, for $t \in[0,0.1]$, the monodromy matrix is in the form

$$
Z(0.1)=\left[\begin{array}{ll}
0.9504317768 & 0.09829249245 \\
-0.9829249236 & 0.9494488518
\end{array}\right]
$$

From monodromy matrix, the multipliers are found as $\rho_{1}=0.9499403143+0.3108277644 i$ and $\rho_{2}=0.9499403143-0.3108277644 i$, so, $\left|\rho_{1}\right|=\left|\rho_{2}\right|=0.99950013<1$. Since unity is not one of the multipliers, the spring-mass system (6.6) has a unique 0.1 -periodic solution with the initial value $\left(z_{1}(0), z_{2}(0)\right)=(-0.001015784822,0.00001018364466)$ evaluated by using the equation (5.1). The simulations showing 0.1 -periodic solution of (6.6) are given in Figure 5. 

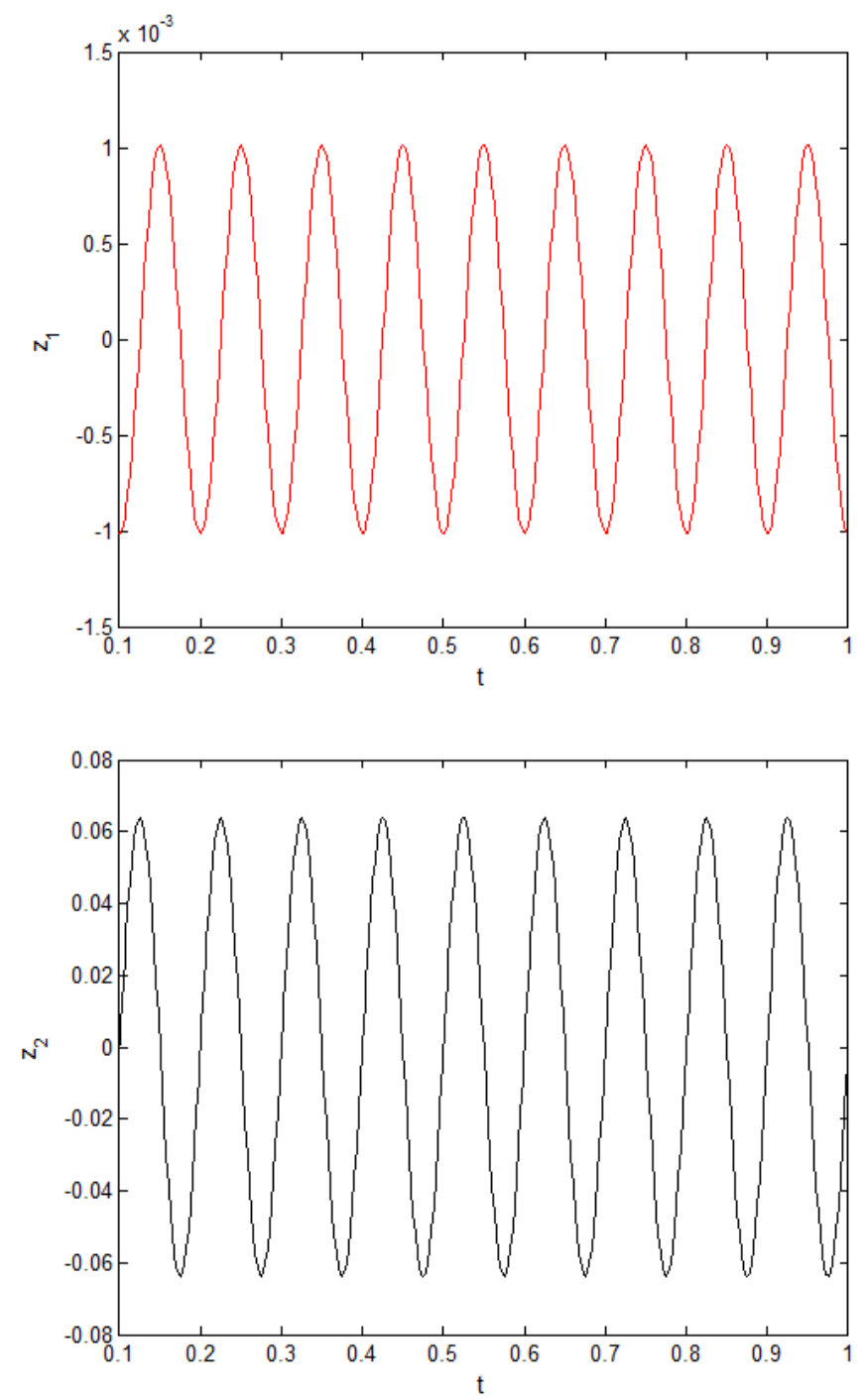

Figure 5. Simulations showing the 0.1 -periodic solutions of (6.6) for $t \in[0,10]$ with $k=10, m=1, c=0.01, A=$ $0.00000001, \theta_{i}=\zeta_{i}=\frac{1}{10} i, i \in \mathbb{Z}$ starting at $\left(z_{1}(0), z_{2}(0)\right)=$ $(-0.001015784822,0.00001018364466)$

Besides, the simulation according to the solutions $z_{1}(t)$ and $z_{2}(t)$ in Figure 6 shows the existence of periodic solution. 


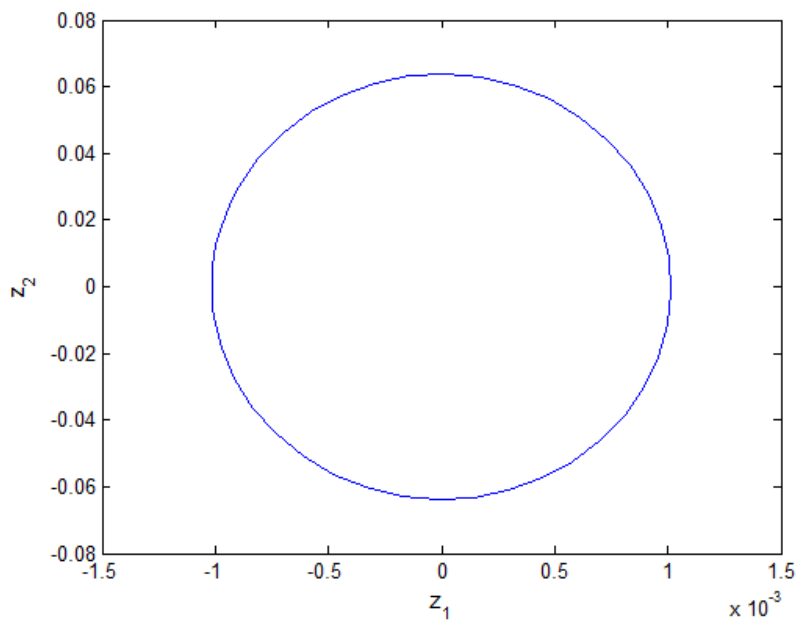

Figure 6. Simulation according to the solutions $z_{1}(t)$ and $z_{2}(t)$ showing the 0.1 -periodic solutions of $(6.6)$ for $t \in[0,10]$ with $k=10$, $m=1, c=0.01, A=0.00000001, \theta_{i}=\zeta_{i}=\frac{1}{10} i, i \in \mathbb{Z}$ starting at $\left(z_{1}(0), z_{2}(0)\right)=(-0.001015784822,0.00001018364466)$

6.4. Example. Let $m=1, c=1, k=100, A=101$ and the sequences $\theta_{i}=\zeta_{i}=\frac{1}{10} i$. In other words, consider the spring mass system

$$
x^{\prime \prime}+x^{\prime}+100 x=101 x(\gamma(t))
$$

Taking $z_{1}=x$ and $z_{2}=x^{\prime}$, spring-mass system (6.7) can be reduced to the following form

$$
z^{\prime}(t)=\left[\begin{array}{cc}
0 & 1 \\
-100 & -1
\end{array}\right] z(t)+\left[\begin{array}{cc}
0 & 0 \\
101 & 0
\end{array}\right] z(\gamma(t))
$$

Since the conditions $(A 1)$ and $(A 3)$ are satisfied, the equation (6.7) and so the system (6.8) are 0.1 -periodic. $t=\omega=0.1 \in\left[\theta_{0}, \theta_{1}\right]=[0,0.1]$. Then, for $t \in[0,0.1]$, the monodromy matrix is in the form

$$
Z(0.1)=\left[\begin{array}{ll}
1.056233890 & 0.08007901070 \\
0.08007901070 & 0.4749127097
\end{array}\right]
$$

Thus, we find the multipliers $\rho_{1}=1.067063311$ and $\rho_{2}=0.4640832890$. So, according to Theorem 5.1., there exists nontrivial solutions $z(t+0.1)=\rho_{1} z(t)$ and $z(t+0.1)=\rho_{2} z(t)$ of the system (6.8). It is seen that the spring-mass system (6.8) has unstable solutions, in particular, the trivial solution of the system (6.8) is unstable. The simulations showing instability of the trivial solution of (6.8) are given in Figure 7. 


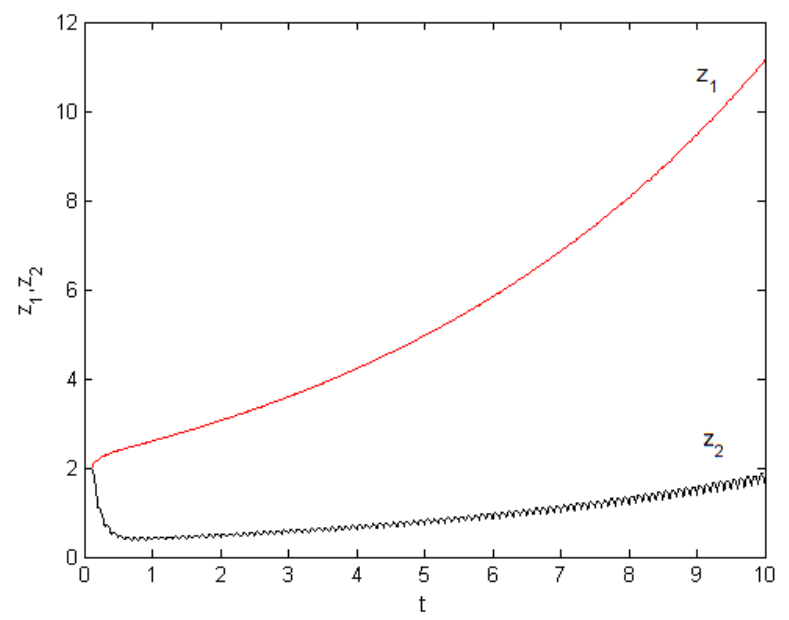

Figure 7. Simulation showing the unstable solutions of (6.8) for $t \in$ $[0,10]$ with $m=1, c=1, k=100, A=101, \theta_{i}=\zeta_{i}=0.1 i, i \in \mathbb{Z}$ starting at $\left(z_{1}(0), z_{2}(0)\right)=(2,2)$

\section{References}

[1] Aftabizadeh, A.R., Wiener, J. and Xu, J.-M. Oscillatory and periodic solutions of delay differential equations with piecewise constant argument, Proc. Amer. Math. Soc. 99, 673$679,1987$.

[2] Akhmet, M.U. Integral manifolds of differential equations with piecewise constant argument of generalized type, Nonlinear Anal. 66, 367-383, 2007.

[3] Akhmet, M.U. Nonlinear Hybrid Continuous/Discrete Time Models, Amsterdam, Paris, Atlantis Press, 2011.

[4] Akhmet, M.U. On the integral manifolds of the differential equations with piecewise constant argument of generalized type, Proceedings of the Conference on Differential and Difference Equations at the Florida Institute of Technology, August 1-5, 2005, Melbourne, Florida, Editors: R.P. Agarval and K. Perera, Hindawi Publishing Corporation, 11-20, 2006.

[5] Akhmet, M.U. Quasilinear retarded differential equations with functional dependence on piecewise constant argument, Communications On Pure And Applied Analysis 13 (2), 929947, 2014.

[6] Akhmet, M.U. Stability of differential equations with piecewise constant arguments of generalized type, Nonlinear Anal. 68, 794-803, 2008.

[7] Akhmet, M.U. and Aruğaslan, D. Lyapunov-Razumikhin method for differential equations with piecewise constant argument, Discrete and Continuous Dynamical Systems, Series A 25 (2), 457-466, 2009.

[8] Akhmet, M.U., Aruğaslan, D. and Liu, X. Permanence of nonautonomous ratio-dependent predator-prey systems with piecewise constant argument of generalized type, Dynamics of Continuous Discrete and Impulsive Systems Series A. Mathematical Analysis 15 (1), 37-51, 2008.

[9] Akhmet, M.U., Aruğaslan, D. and Yılmaz, E. Stability analysis of recurrent neural networks with piecewise constant argument of generalized type, Neural Networks 23, 805-811, 2010.

[10] Akhmet, M.U., Aruğaslan, D. and Yılmaz, E. Stability in cellular neural networks with a piecewise constant argument, Journal of Computational and Applied Mathematics 233, 2365-2373, 2010. 
[11] Akhmet, M.U., Öktem, H., Pickl, S.W. and Weber, G.-W. An anticipatory extension of Malthusian model, CASYS'05-Seventh International Conference, AIP Conference Proceedings 839, 260-264, 2006.

[12] Busenberg, S. and Cooke, K.L. Models of vertically transmitted diseases with sequentialcontinuous dynamics, Nonlinear Phenomena in Mathematical Sciences, Academic Press, New York, 179-187, 1982.

[13] Chiu, K.-S., Pinto, M. Periodic solutions of differential equations with a general piecewise constant argument and applications, Electron. J. Qual. Theory Differ. Equ. 46, 19 pp, 2010.

[14] Chiu, K.-S., Pinto, M. Variation of parameters formula and Gronwall inequality for differential equations with a general piecewise constant argument, Acta Math. Appl. Sin. Engl. Ser. 27 (4), 561-568, 2011.

[15] Cooke, K.L. and Wiener, J. Retarded differential equations with piecewise constant delays, J. Math. Anal. Appl. 99, 265-297, 1984.

[16] Dai, L. and Singh, M.C. On oscillatory motion of spring-mass systems subjected to piecewise constant forces, Journal of Sound and Vibration 173 (2), 217-231, 1994.

[17] Gopalsamy, K. Stability and Oscillation in Delay Differential Equations of Population Dynamics, Kluwer Academic Publishers, Dordrecht, 1992.

[18] Györi, I. On approximation of the solutions of delay differential equations by using piecewise constant argument, Int. J., Math. Math. Sci., 14, 111-126, (1991).

[19] Gopalsamy, K. and Liu, P. Persistence and global stability in a population model, J. Math. Anal. Appl. 224, 59-80, 1998.

[20] Gyori, I. and Ladas, G. Oscillation Theory of Delay Differential Equations with Applications, Oxford University Press, New York, 1991.

[21] Matsunaga, H., Hara, T. and Sakata, S. Global attractivity for a logistic equation with piecewise constant argument, Nonlinear Differential Equations Appl. 8, 45-52, 2001.

[22] Muroya, Y. Persistence, contractivity and global stability in logistic equations with piecewise constant delays, J. Math. Anal. Appl. 270, 602-635, 2002.

[23] Papaschinopoulos, G. On the integral manifold for a system of differential equations with piecewise constant argument, J. Math. Anal. Appl. 201, 75-90, 1996.

[24] Seifert, G. Almost periodic solutions of certain differential equations with piecewise constant delays and almost periodic time dependence, J. Differential Equations 164, 451-458, 2000.

[25] Shah, S.M. and Wiener, J. Advanced differential equations with piecewise constant argument deviations, Int. J. Math. Math. Sci. 6, 671-703, 1983.

[26] Shen, J.H. and Stavroulakis, I.P. Oscillatory and nonoscillatory delay equation with piecewise constant argument, J. Math. Anal. Appl. 248, 385-401, 2000.

[27] Wang, G. Periodic solutions of a neutral differential equation with piecewise constant arguments, J. Math. Anal. Appl. 326, 736-747, 2007.

[28] Wang, Z. and $\mathrm{Wu}, \mathrm{J}$. The stability in a logistic equation with piecewise constant arguments, Differential Equations Dynam. Systems 14, 179-193, 2006.

[29] Wiener, J. Generalized Solutions of Functional Differential Equations, World Scientific, Singapore, 1993.

[30] Wiener, J. and Cooke, K.L. Oscillations in systems of differential equations with piecewise constant argument, J. Math. Anal. Appl. 137, 221-239, 1989.

[31] Wiener, J. and Lakshmikantham, V. A damped oscillator with piecewise constant time delay, Nonlinear Stud. 7, 78-84, 2000.

[32] Xia, Y., Huang, Z. and Han, M. Existence of almost periodic solutions for forced perturbed systems with piecewise constant argument, J. Math. Anal. Appl. 333, 798-816, 2007.

[33] Yang, X. Existence and exponential stability of almost periodic solution for cellular neural networks with piecewise constant argument, Acta Math. Appl. Sin. 29, 789-800, 2006. 